\title{
DNA methylation profiling of synovial fluid-derived FLS in rheumatoid arthritis reveals common changes relative to tissue-derived FLS
}

John R. Glossop ${ }^{1,2}$, Kim E. Haworth ${ }^{1}$, Richard D. Emes ${ }^{3,4}$, Nicola B. Nixon ${ }^{2}$, Jon C.

Packham $^{2}$, Peter T. Dawes ${ }^{2}$, Anthony A. Fryer ${ }^{1}$, Derek L. Mattey ${ }^{1,2}$, William E. Farrell ${ }^{1}$

${ }^{1}$ Institute for Science and Technology in Medicine, Keele University, Guy Hilton

Research Centre, Thornburrow Drive, Hartshill, Stoke-on-Trent, Staffordshire, ST4 7QB, UK.

${ }^{2}$ Haywood Rheumatology Centre, Haywood Hospital, High Lane, Burslem, Stoke-onTrent, Staffordshire, ST6 7AG, UK.

${ }^{3}$ School of Veterinary Medicine and Science, University of Nottingham, Sutton Bonington Campus, Sutton Bonington, Leicestershire, LE12 5RD, UK.

${ }^{4}$ Advanced Data Analysis Centre, University of Nottingham, Sutton Bonington Campus, Sutton Bonington, Leicestershire, LE12 5RD, UK.

Author for correspondence/reprints: Dr. John. R. Glossop, Institute for Science and Technology in Medicine, Keele University, Haywood Rheumatology Centre, Haywood Hospital, High Lane, Burslem, Stoke-on-Trent, Staffordshire, ST6 7AG, UK.

Tel: $+44(0) 1782673812$

Fax: +44 (0) 1782673509

E-mail: j.r.glossop@keele.ac.uk 
Key words: Rheumatoid arthritis; DNA Methylation; Fibroblast-like synoviocyte; Synovial fluid; Epigenetics; Osteoarthritis.

Funding support: This work was supported by funding provided by the Haywood Rheumatism Research and Development Foundation.

Running footline: DNA methylation in RA fluid-derived FLS 


\section{Abstract}

Aims. Alterations in DNA methylation contribute to the abnormal phenotype of fibroblast-like synoviocytes (FLS) from patients with rheumatoid arthritis (RA). Herein, we performed genome-wide DNA methylation profiling of RA FLS derived from synovial fluid, a more readily accessible source of disease-associated cells.

Patients \& Methods. Synovial fluid-derived FLS were isolated and expanded in vitro from 12 patients with RA and osteoarthritis (OA). DNA methylation was interrogated using HumanMethylation450 BeadChips and bisulfite pyrosequencing.

Results. Array analysis identified 328 CpGs, representing 195 genes, that were differentially methylated between RA and OA fluid-derived FLS. Comparison with the genes identified in two independent studies of tissue-derived FLS revealed 73 genes in common ( 40\%), of which 22 shared identity with both studies. Pyrosequencing confirmed altered methylation of these genes.

Conclusions. Synovial fluid-derived RA FLS show methylation changes across multiple genes. A subset of these are common with tissue-derived FLS, supporting the use of fluid-derived FLS for future investigations. 


\section{Introduction}

Rheumatoid arthritis (RA) is an autoimmune inflammatory disease of unknown etiology. The hallmark of the disease is synovial hyperplasia, chronic inflammation and progressive destruction of the synovial joints. Fibroblast-like synoviocytes (FLS), one of the constituent cells of the synovium which envelops the joint, represent one of the principal effectors of joint damage in RA [1]. Indeed, these cells are a key component of the hyperplastic, inflamed and invasive synovial tissue mass that extends from the RA synovium and which promotes persistent degradation of articular cartilage and bone.

In contrast to normal synovial FLS, these cells in patients with RA display an activated, aggressive and invasive phenotype, with characteristics of transformed cells [2, 3]. Other features of the activated phenotype include increased resistance to apoptosis and expression of a myriad of inflammatory mediators, adhesion molecules and matrixdegrading enzymes that coordinately support local inflammation and the destructive process $[1,4,5]$. The factors that contribute to the abnormal FLS phenotype apparent in RA are not completely understood. However, emerging data supports an important role for changes to the epigenomic landscape in this process $[6,7]$.

In disparate diseases, the principal epigenetic mechanisms that are subject to inappropriate reprogramming include DNA methylation, histone modifications and expression of non-coding RNAs that include microRNAs (miRNAs). These modifications frequently impact on gene expression or translational efficiency, and in the context of methylation at promoter-associated CpGs, are frequently associated with transcriptional repression [8]. RA-derived FLS display global changes in levels of DNA 
methylation and experimentally-induced global demethylation promotes the transition of normal synovial fibroblasts to an RA fibroblast-like phenotype [9]. Gene-specific methylation changes are also apparent in RA-derived fibroblasts. In this context, aberrant methylation has been described for genes such as the apoptosis-related death receptor 3 [10], the miRNA miR-124a [11], implicated in the proliferation of RA fibroblasts [12], and for chemokine and TGF- $\beta$ signalling genes [13, 14]. Moreover, recent genome-wide studies have reported hypomethylation and hypermethylation of multiple genes in tissue-derived FLS from patients with RA [14-16].

Synovial tissue is the principal source of FLS. However, these samples require invasive surgery and are often limited in number making their study technically challenging. In contrast, synovial fluid represents a more readily accessible source of FLS. Moreover, studies of these cells in RA have revealed morphological and phenotypic characteristics consistent with those apparent in their tissue-derived counterparts [17-19], suggestive of a role in joint pathology. In this work, we examine for the first time the epigenetic profile of synovial fluid-derived FLS by interrogating genome-wide DNA methylation in these cells from patients with RA and relative to similar cells from patients with osteoarthritis (OA). Since two recent studies $[15,16]$ have performed broadly similar analyses using the same array platform in tissue-derived FLS, we have also been able to appraise our findings relative to these reports to determine similarities and differences in methylation between fluid-derived FLS and their tissue-derived counterparts. 


\section{Patients \& Methods}

\section{Patients}

Genome-wide DNA methylation was studied in synovial fluid-derived FLS from six female Caucasian patients with RA and six patients with OA. RA patients satisfied the 1987 American College of Rheumatology revised criteria set [20] and had established disease $(14.1 \pm 6.3$ years duration, mean \pm SD). Patients had been treated with methotrexate $(n=4)$, sulphasalazine $(n=5)$ and/or gold salts $(n=3)$, and two were rheumatoid factor positive (data on anti-citrullinated peptide antibody status were not available for this cohort). Inflammatory markers (ESR) were within the normal range in four patients and were raised in two patients. OA patients, diagnosed by a consultant rheumatologist based on clinical findings, all had recent-onset disease (duration $<3$ months) and were recruited as a non-inflammatory control group in which the impact of disease was minimal. The average ages of the RA and OA groups was $68.7 \pm 11.2$ and $66.9 \pm 9.1$ years, respectively (mean \pm SD). All patients were recruited at the Haywood Rheumatology Centre in Stoke-on-Trent, UK and gave written informed consent. The study was approved by the East Midlands (Derby) Research Ethics Committee.

\section{Culture of synovial fluid-derived FLS}

Synovial fluid samples obtained during routine knee arthrocentesis were processed within 1 hour of collection. Cells were pelleted and resuspended in Quantumm-333 fibroblast culture medium (PAA Laboratories, GE Healthcare) in $25 \mathrm{~cm}^{2}$ tissue-culture flasks (PAA Laboratories). Non-adherent cells were aspirated following overnight incubation. Adherent cultures (fibroblasts and some macrophage-like cells) were then serially passaged and FLS isolated using trypsinization procedures that we and others 
have previously described [17-19,21], and in which FLS readily detach whilst non-FLS cells remain resistant to trypsinization. Using this approach, homogeneous cultures of adherent FLS were achieved by three serial passages, as confirmed by light microscopy. Cell cultures were maintained at $37^{\circ} \mathrm{C}$ in a $5 \% \mathrm{CO}_{2}$ humidified atmosphere.

\section{Genomic DNA extraction and sodium bisulfite conversion}

Passage three, fluid-derived FLS were used in all cases. Genomic DNA was extracted from homogenized cell lysates (QIAShredder spin columns; Qiagen) using a spin column-based format (AllPrep DNA/RNA Mini kit; Qiagen). Genomic DNA was further purified and concentrated by ethanol-based precipitation reactions, and assessed on a NanoDrop ND-1000 spectrophotometer (Thermo Scientific). Genomic DNA underwent sodium bisulfite conversion using an EZ DNA Methylation Kit performed as recommended by the manufacturer (Zymo Research).

\section{Genome-wide DNA methylation profiling by HumanMethylation450 BeadChip}

Genome-wide DNA methylation was interrogated at over 480,000 individual CpG dinucleotides using an Infinium HumanMethylation450 BeadChip (henceforth referred to as " $450 \mathrm{~K}$ array" or "array"; Illumina Inc.). Array design and coverage has been described elsewhere [22]. Sample preparation and array hybridization and processing was performed by Gen-Probe Life Sciences Ltd (Manchester, UK), according to Illumina recommended protocols that we have described previously [23]. A single sample failed the manufacturer's robust internal array quality control assessment and on this basis the sample was removed from the study and prior to data analysis. 
Methylation at individual CpGs is reported as a methylation $\beta$-value [22], which fall on a continuous linear scale ranging from 0 (unmethylated) to 1 (completely methylated).

\section{Sodium bisulfite pyrosequencing}

Independent validation of array-identified candidates was performed using sodium bisulfite pyrosequencing. Prior to this, and to increase template quantity, we conducted whole genome amplification of bisulfite converted genomic DNA, as described previously [23]. Pyrosequencing assays were designed using PyroMark Assay Design software (v 2.0.1.15, Qiagen) and primer sets were purchased from Biomers.net or Invitrogen/Life Technologies. PCR amplicons encompassing CpGs of interest were prepared using a touchdown protocol, details of which we have recently described [24]. Pyrosequencing assays included one or more control dispensations to confirm sequence identity and completeness of bisulfite conversion, and were run on a PyroMark Q24 instrument (Qiagen) according to manufacturer recommendations. Assay details are provided in Supplementary Table 1. Pyrograms were analyzed using PyroMark Q24 software (v 2.0.6., build 20; Qiagen).

\section{Data analysis}

Array data $(\mathrm{n}=11$, five RA and six OA) were processed and analyzed using NIMBL software [25] and passed initial quality control based on the performance of internal array controls. We excluded all CpGs for which one or more of the 11 samples had $\beta$ value detection $p$-values $>0.05$, and sites with at least one missing $\beta$-value. The processed dataset, which consisted of 468,961 CpGs (from a total of 482,421 array CpG sites [22]), was adjusted for the reported difference in sensitivity between the two 
Infinium probe types by performing 'peak-based correction' [26]. To identify CpGs/genes differentially methylated between RA and OA-derived FLS, we performed a series of filtering steps (see Results). We elected to use a minimum $\beta$-value difference between the RA and OA groups of 0.1 , in part to permit comparisons with two recent studies of tissue-derived FLS also using the $450 \mathrm{~K}$ array platform $[15,16]$.

Coordinated patterns of methylation across RA and OA-derived FLS were examined by hierarchical clustering using Genesis (v1.7.6) [27], with Euclidian distance and average linkage criteria. Genes associated with differentially methylated CpGs were assessed for enrichment of gene ontology terms and biological pathways using DAVID online software, where statistically significant ontology terms/pathways were those with a pvalue $<0.05$ calculated using the EASE score $[28,29]$. The false discovery rate (FDR) [30] was used to adjust for multiple testing. 


\section{Results}

\section{Genome-wide DNA methylation in RA fluid-derived FLS}

We performed genome-wide analysis of DNA methylation in RA and OA fluid-derived FLS using an Infinium-based 450K array that examines over 480,000 individual CpG sites. Subsequent to initial data processing, we used a series of stringent filtering criteria to identify sites displaying altered methylation between RA and OA FLS (Figure 1). We considered a $\mathrm{CpG}$ to be differentially methylated if the mean $\beta$-value difference between the RA and OA groups was $\geq 0.1$ and was statistically significant following a FDR-adjusted t-test. Using this approach we identified 328 unique CpGs that were differentially methylated between fluid-derived FLS from RA and OA patients. Similar results were obtained when we performed a technical replicate on a second array (data not shown). This, combined with additional independent validation of candidate sites (described in a subsequent section), reinforces the robustness of the array dataset.

Of the 328 CpGs identified, the majority of sites were hypomethylated in RA (258 CpGs; 78.7\%). Approximately two-thirds of the sites demonstrated a mean $\beta$-value difference of at least 0.25 between the groups $(205 ; 62.5 \%)$, and in some cases the differences were particularly pronounced ( $\beta$-value differences up to 0.72 ). The altered methylation observed in RA FLS was in fact evident for the majority of the individual samples examined. As example, for two-thirds of the $328 \mathrm{CpG}$ sites, four out of five RA samples displayed methylation $\beta$-value differences that were at least 0.2 greater/lower than the average methylation in OA FLS. The 328 differentially methylated CpGs represented a total of 195 individual genes. A complete list and annotation for the 328 identified sites is presented in Supplementary Table 2. 
To determine whether the methylation patterns across these 328 sites discriminated between FLS from RA and OA patients, we performed hierarchical clustering. The heatmap in Figure 2 shows that RA and OA-derived FLS possess intrinsically distinct methylation patterns across these sites. Moreover, this analysis also shows that there was negligible inter-individual variation within each of the patient groups. However, an exception to this finding was apparent for one OA sample which clustered with the RA group. Gene ontology and pathway enrichment analysis for the 195 genes using DAVID $[28,29]$ identified multiple overrepresented ontology terms (e.g. relating to proteoglycan synthesis, catabolic processes). However, enrichment of these terms was not statistically significant following adjustment for multiple comparisons.

\section{Fluid-derived FLS display methylation changes both common with and unique from tissue-derived FLS}

We next analyzed the 195 genes identified as differentially methylated in fluid-derived FLS (Figure 1) relative to recent findings describing DNA methylation in tissuederived cells. In these cases, Nakano et al [15] and de la Rica et al [16], also using Illumina 450K arrays, examined genome-wide DNA methylation in tissue-derived FLS from RA and OA patients. In both these reports and our own study, the selection criteria were broadly similar, requiring a minimum $\beta$-value difference of 0.1 in combination with an adjusted t-test to identify differentially methylated CpGs. Genes represented by differentially methylated sites in each of the published studies were obtained from supplementary data accompanying each article (for comparison purposes, mean differences were calculated for the CpGs reported by de la Rica and colleagues [16]). 
As shown in the Venn-diagram in Figure 3, comparison of the 195 genes identified as differentially methylated in our study with those reported by Nakano et al [15] and de la Rica et al [16] identified 50 genes and 45 genes that were common with and significantly associated with each study, respectively $\left(\mathrm{p}<1.0 \times 10^{-14}\right.$; Figure 3). In total, 73 genes $(\sim 40 \%)$ shared identity with at least one of the two studies, and of which 22 genes were common to all three genome-wide studies (details are provided in Table 1). For 18 of these 22 genes, at least four of the five individual RA FLS samples displayed methylation $\beta$-value changes at least 0.2 greater/lower than the average methylation observed in OA-derived FLS. Heatmap analysis of the 22 genes revealed clear differences in the pattern of methylation between RA and OA-derived FLS (Figure 3). However, one OA sample, and consistent with clustering for the original $328 \mathrm{CpGs}$, was again found to cluster with the RA group. Moreover, for seven of the 22 genes, the direction of change in methylation was the same in all three studies (hypomethylation for 6 genes and hypermethylation for 1 gene). These seven genes were C18orf45, LMO4, MAP3K5, ODZ4, PKNOX2, PRDM16 and SEPT11 (indicated by bold font in Table 1). For the remaining 15 genes (with two exceptions), agreement in the context of the direction of the methylation change (hypo- or hypermethylation) was apparent in our study and in one of the two studies examining tissue-derived FLS.

In addition to the genes described above, our array analyses also identified 122 differentially methylated genes that are unique to our study of fluid-derived FLS (gene details are provided in Supplementary Table 3). Hierarchical clustering of these genes revealed distinct methylation patterns between RA and OA-derived FLS, whereby 
samples segregated into two distinct clusters according to diagnosis (Supplementary

Figure 1). Gene ontology and pathway enrichment analysis of these genes did not identify any statistically significant overrepresented terms (following FDR adjustment).

Amongst the genes that were hypomethylated and unique to RA fluid-derived FLS (relative to OA), the largest $\beta$-value difference observed (0.72) was in MSRA, a gene coding for a methionine sulfoxide reductase involved in the response to oxidative stress. Hypomethylation was also observed for a number of other genes relevant to RA, such as the metalloproteinase ADAMTS14 (an extracellular matrix degrading enzyme; $\beta$-value difference 0.36 ), and $M I R 155 H G$, the host gene coding for the immuno-regulatory microRNA miR-155 (0.34).

\section{Candidate gene validation by pyrosequencing}

For independent validation of the genome-wide array data, we performed bisulfite pyrosequencing for a number of differentially methylated candidate genes/CpGs. Validation was conducted across a total of 17 samples, comprising six OA and 11 RA samples, and which included a total of eight independent samples (one OA and seven RA). We selected the seven genes (C18orf45, LMO4, MAP3K5, ODZ4, PKNOX2, PRDM16 and SEPT11) for which methylation changes were observed in the same direction (hypomethylation or hypermethylation) in our study and each of the two studies of tissue-derived FLS $[15,16]$. A further four genes that were unique to our study of fluid-derived FLS (ADAMTS14, MIR155HG, RUNX1 and ULK3) were also examined (see Supplementary Table 1 for assay details). 
Array $\beta$-values and methylation levels determined by pyrosequencing for candidate genes are presented in Figure 4. In each case, the statistically significant methylation differences observed between RA and OA-derived FLS by array analysis were reproduced by pyrosequencing ( $S E P T 11$ is not shown since an assay incorporating the array-interrogated $\mathrm{CpG}$ site could not be designed). For some genes, for example $M A P 3 K 5$ and $R U N X 1$, pyrosequencing analysis also facilitated interrogation of additional CpGs adjacent to the array identified site. In these cases, the data showed altered methylation that was consistent with the changes at the array site (one site for $M A P 3 K 5$, and two sites for $R U N X 1$; Figure 5). As has been reported previously by ourselves and other investigators [23,31], methylation levels determined by pyrosequencing were generally lower than their respective array $\beta$-values. The robustness of our array data through validation of multiple candidates is exemplified by the strong positive correlation between array $\beta$-values and methylation determined by pyrosequencing (Spearman's r = 0.955; Supplementary Figure 2). 


\section{Discussion}

Synovial tissue from patients with RA is typified by hyperplasia of the lining layer and invasion of the articular cartilage and bone. FLS, one of two specialized cell types comprising the lining layer, display an activated, aggressive and invasive phenotype [1, $4,5]$ and play a key role in the destructive process, supporting these cells as a potential therapeutic target in RA [32]. The origin of FLS within the synovial fluid remains unclear, but derivation from the tissue itself or from progenitor cells within the fluid are possibilities $[17,33]$. Studies of these fluid-derived cells indicate morphological and phenotypic characteristics consistent with their tissue-derived counterparts, including the capacity to invade and degrade cartilage [17-19]. Epigenetic modifications in rheumatoid FLS are important [9-16], but to our knowledge there have been no reports describing DNA methylation in fluid-derived FLS. Using high resolution DNA methylation arrays, we are, to our knowledge, the first to describe genome-wide methylation changes in fluid-derived FLS in RA. Moreover, we have identified subsets of genes for which the methylation changes in fluid-derived FLS are either common to or distinct from genes previously identified on the same array platform in tissue-derived FLS $[15,16]$. Our findings, if confirmed by other investigators, would support the use of FLS derived from synovial fluid as a more readily accessible and available source of material for future investigations of RA synovial fibroblasts in the disease process and perhaps for clinical management.

We identified a total of 195 genes which displayed statistically significant methylation changes in fluid-derived FLS from patients with RA. A key finding of our study was the identification of 73 genes, approximately $40 \%$ of our gene-set, which have also been 
identified as aberrantly methylated in two previous genome-wide investigations of tissue-derived FLS $[15,16]$. Moreover, of these, a subset of 22 differentially methylated genes displayed altered methylation in all three studies. Most notably, seven of the genes displayed changes in methylation in RA fluid-derived FLS (for at least four of the five individual samples) that were consistent with respect to the direction of change reported for tissue-derived FLS (hypomethylation for six genes and hypermethylation for one gene). Identification of these candidates in our study, and in two independent studies that pre-date our report, supports an important role in the RA synovial fibroblast phenotype and associated joint pathology. Moreover, these findings are compelling for shared, inappropriate, epigenetic modification in RA FLS irrespective of their origin. For one of the genes, $M A P 3 K 5$, constitutive expression has been shown to promote arthritis and synovial fibroblast proliferation [34], whilst suppression inhibits IL-6 expression and attenuates joint damage [35]. Evidence also supports regulation of MAP3K5 expression in activated FLS through reduced expression of specific miRNAs [36], highlighting a role for epigenetic control in these cells. Our data now suggest that this may also extend to include aberrant hypomethylation of the MAP3K5 gene.

The comparative analysis we conducted between fluid-derived and tissue-derived FLS also revealed a significant number of differentially methylated genes that were unique to our study of fluid-derived FLS. This included hypomethylation of multiple genes, such as $A D A M T S 14$, a member of the ADAMTS (a disintegrin and metalloproteinase with thrombospondin motifs) family of proteinases implicated in RA [37], and MSRA, an enzyme involved in the response to oxidative damage and for which a recent genetic association with RA has been described [38]. Of particular note, we detected 
hypomethylation of $M I R 155 H G$, host gene of the miRNA miR-155 which has previously been implicated in arthritis. Indeed, studies have demonstrated elevated expression of miR-155 in RA, including synovial fibroblasts and synovial fluid-derived cells [39-41], and support an essential regulatory role for miR-155 in animal models of arthritis and autoimmunity [41-43]. By array and pyrosequencing analysis, we observed aberrant hypomethylation of $M I R 155 H G$ in RA fluid-derived FLS, suggesting a possible role in the regulation of miR-155. Although we are not aware of any prior studies describing the methylation status of the miR-155 gene in RA, studies in cancer show methylation-dependent regulation of this and other miRNAs [44, 45]. It is unclear at this time the reason for the disparity between $M I R 155 H G$ methylation changes observed in fluid-derived but not tissue-derived FLS $[15,16]$. Functional studies are required to explore the impact of $M I R 155 H G$ hypomethylation in RA synovial cells.

The majority of differentially methylated sites identified in RA FLS were hypomethylated (78.7\%) and in some cases the methylation changes were substantial ( $\beta$-value differences up to 0.72 ). Notably, and as we recently observed for diseasespecific methylation changes in RA-derived T- and B-cell populations (unpublished observations), the majority of differentially methylated CpGs were located within the body of genes (intragenic). Indeed, these finding are consonant with the differential methylation patterns previously identified in tissue-derived FLS from patients with RA, where altered gene-body methylation was a common finding $[15,16]$. Although not specifically addressed in this or previous reports, other studies have described associations between gene-body methylation patterns and gene expression [46-48]. In 
these cases, hypomethylation in RA synovial fibroblasts may well influence gene expression in both fluid- and tissue-derived cells, and warrants further investigation.

The candidate genes identified in this study were on the basis of stringent filtering criteria. We made comparisons with two recent genome-wide studies that used broadly similar criteria, but in these cases of tissue-derived FLS $[15,16]$. We are mindful that our own study and those that interrogated tissue-derived FLS represent distinct patient cohorts using limited sample numbers. These limitations may, in part, explain differences in the absolute numbers of CpGs/genes identified. Encouragingly however, commonality was apparent for 73 genes $(\sim 40 \%)$ across the three independent studies. Together with validation of our data using an independent technique we are confident that the identification of candidate genes in RA-derived FLS is founded on robust methods. It will remain important however for these candidates to be verified, and the consequences of differential methylation established, in larger patient cohorts. 


\section{Conclusions}

We provide the first report of genome-wide changes in DNA methylation in FLS derived from the synovial fluid of patients with RA. A subset of candidate genes were identified and validated in fluid-derived FLS that were common with genes described in independent studies of tissue-derived FLS. These data support an important role for these genes in the abnormal disease-associated phenotype of RA FLS and demonstrate that epigenetic changes in synovial tissue are, at least in part, recapitulated in fluidderived cells. Our findings also indicate a role for unique methylation changes in fluidderived FLS and support the use of synovial fluid as a viable and more readily available alternative for the study epigenetic dysregulation in RA synovial fibroblasts. 


\section{Executive Summary}

\section{Background}

- Increasing evidence indicates that changes to the DNA methylome contribute to the abnormal phenotype of FLS from patients with RA.

- Synovial tissue is the principal source of RA FLS, although the limited number of these samples makes their study technically challenging. FLS are also present in the synovial fluid, a more readily accessible source of these cells.

- This study examined genome-wide DNA methylation in synovial fluid-derived FLS from patients with RA.

\section{Patients \& Methods}

- Genome-wide DNA methylation was examined in synovial fluid-derived, passage three FLS from six RA and six OA patients using the HumanMethylation450 BeadChip.

- Differentially methylated genes were appraised relative to genes previously reported in two independent genome-wide studies of RA tissue-derived FLS. Validation of candidate genes was performed by bisulfite pyrosequencing $(n=17)$.

\section{Results}

- $\quad$ Significant changes in DNA methylation were identified for 195 genes (328 CpGs) in fluid-derived FLS from patients with RA (relative to FLS from OA patients).

- A significant proportion ( $\sim 40 \%)$ of these genes were common with those previously reported in two recent studies of tissue-derived FLS, and included 22 genes common to all three studies.

- Two of these candidates, MAP3K5 and MIR155HG, have previously been shown to play an important role in the pathogenesis of arthritis. 


\section{Conclusions}

- These findings identify a number of candidate genes and support an important role for epigenetic control in the abnormal FLS phenotype observed in RA.

- Moreover, our findings demonstrate support the use of FLS derived from synovial fluid as a viable and more accessible source of FLS for future investigations.

- Such work should include analysis of paired synovial tissue and synovial fluid samples to further explore epigenetic dysregulation in RA synovial fibroblasts. 


\section{References}

Papers of interest have been highlighted as:

* of interest

1. Bartok B, Firestein GS. Fibroblast-like synoviocytes: key effector cells in rheumatoid arthritis. Immunol. Rev. 233(1), 233-255 (2010).

2. Lafyatis R, Remmers EF, Roberts AB, Yocum DE, Sporn MB, Wilder RL. Anchorage-independent growth of synoviocytes from arthritic and normal joints: stimulation by exogenous platelet-derived growth factor and inhibition by transforming growth factor- $\beta$ and retinoids. J. Clin. Invest. 83(4), 1267-1276 (1989).

3. Kinne RW, Boehm S, Iftner T, et al. Synovial fibroblast-like cells strongly express jun-B and c-fos proto-oncogenes in rheumatoid- and osteoarthritis. Scand. J. Rheumatol. Suppl. 101, 121-125 (1995).

4. Ospelt C, Neidhart M, Gay RE, Gay S. Synovial activation in rheumatoid arthritis. Front. Biosci. 9, 2323-2334 (2004).

5. Neumann E, Lefevre S, Zimmermann B, Gay S, Muller-Ladner U. Rheumatoid arthritis progression mediated by activated synovial fibroblasts. Trends. Mol. Med. 16(10), 458-468 (2010).

6. Karouzakis E, Gay RE, Gay S, Neidhart M. Epigenetic control in rheumatoid arthritis synovial fibroblasts. Nat. Rev. Rheumatol. 5(5), 266-272 (2009).

7. Klein K, Gay S. Epigenetic modifications in rheumatoid arthritis, a review. Curr. Opin. Pharmacol. 13(3), 420-425 (2013).

8. Bird A. DNA methylation patterns and epigenetic memory. Genes Dev. 16(1), 6-21 (2002). 
9. Karouzakis E, Gay RE, Michel BA, Gay S, Neidhart M. DNA hypomethylation in rheumatoid arthritis synovial fibroblasts. Arthritis Rheum. 60(12), 3613-3622 (2009).

10. Takami N, Osawa K, Miura Y, et al. Hypermethylated promoter region of DR3, the death receptor 3 gene, in rheumatoid arthritis synovial cells. Arthritis Rheum. 54(3), 779-787 (2006).

11. Zhou Q, Long L, Shi G, Zhang J, Wu T, Zhou B. Research of the methylation status of miR-124a gene promoter among rheumatoid arthritis patients. Clin. Dev. Immunol. 2013, 524204 (2013).

12. Nakamachi Y, Kawano S, Takenokuchi M, et al. MicroRNA-124a is a key regulator of proliferation and monocyte chemoattractant protein 1 secretion in fibroblast-like synoviocytes from patients with rheumatoid arthritis. Arthritis Rheum. 60(5), 12941304 (2009).

13. Karouzakis E, Rengel Y, Jungel A, et al. DNA methylation regulates the expression of CXCL12 in rheumatoid arthritis synovial fibroblasts. Genes Immun. 12(8), 643$652(2011)$

14. Park SH, Kim SK, Choe JY, et al. Hypermethylation of EBF3 and IRX1 genes in synovial fibroblasts of patients with rheumatoid arthritis. Mol. Cells. 35(4), 298-304 (2013).

15. Nakano K, Whitaker JW, Boyle DL, Wang W, Firestein GS. DNA methylome signature in rheumatoid arthritis. Ann. Rheum. Dis. 72(1), 110-117 (2013).

*First genome-wide study of DNA methylation in rheumatoid arthritis FLS using the Illumina HumanMethylation450 array. 
16. de la Rica L, Urquiza JM, Gomez-Cabrero D, et al. Identification of novel markers in rheumatoid arthritis through the integrated analysis of DNA methylation and microRNA expression. J. Autoimmun. 41, 6-16 (2013).

*Study reporting the genome-wide relationship between DNA methylation and microRNA expression in RA FLS.

17. Neidhart M, Seemayer CA, Hummel KM, Michel BA, Gay RE, Gay S. Functional characterization of adherent synovial fluid cells in rheumatoid arthritis: destructive potential in vitro and in vivo. Arthritis Rheum. 48(7), 1873-1880 (2003).

\section{*Demonstration of the similar disease-associated characteristics of synovial}

\section{fluid-derived FLS to these cells derived from synovial tissue.}

18. Stebulis JA, Rossetti RG, Atez FJ, Zurier RB. Fibroblast-like synovial cells derived from synovial fluid. J. Rheumatol. 32(2), 301-306 (2005).

19. Ahn JK, Kim H, Lee J, Bee EK, Cha HS, Koh EM. Phenotypic characterization and invasive properties of synovial fluid-derived adherent cells in rheumatoid arthritis. Inflammation 31(6), 365-371 (2008).

20. Arnett FC, Edworthy SM, Bloch DA, et al. American Rheumatism Association 1987 revised criteria for the classification of rheumatoid arthritis. Arthritis Rheum. 31(3), 315-324 (1988).

21. Mattey DL, Dawes PT, Nixon NB, Slater H. Transforming growth factor B1 and interleukin 4 induced a smooth muscle actin expression and myofibroblast-like differentiation in human synovial fibroblasts in vitro: modulation by basic fibroblast growth factor. Ann. Rheum. Dis. 56(7), 426-431 (1997).

22. Bibikova M, Barnes B, Tsan C, et al. High density DNA methylation array with single CpG resolution. Genomics 98(4), 288-295 (2011). 
23. Glossop JR, Nixon NB, Emes RD, et al. Epigenome-wide profiling identifies significant differences in DNA methylation between matched-pairs of T- and Blymphocytes from healthy individuals. Epigenetics 8(11), 1188-1197 (2013).

24. Haworth KE, Farrell WE, Emes RD, et al. Combined influence of gene-specific cord blood methylation and maternal smoking habit on birth weight. Epigenomics 5(1), 37-49 (2013).

25. Wessely F, Emes RD. Identification of DNA methylation biomarkers from Infinium arrays. Front. Genet. 3, 161 (2012).

26. Dedeurwaerder S, Defrance M, Calonne E, Denis H, Sotiriou C, Fuks F. Evaluation of the Infinium Methylation 450K technology. Epigenomics 3(6), 771-784 (2011).

27. Sturn A, Quackenbush J, Trajanoski Z. Genesis: cluster analysis of microarray data. Bioinformatics 18(1), 207-208 (2002).

28. Huang da W, Sherman BT, Lempicki RA. Systematic and integrative analysis of large gene lists using DAVID Bioinformatics Resources. Nature Protoc. 4(1), 44-57 (2009).

29. Huang da W, Sherman BT, Lempicki RA. Bioinformatics enrichment tools: paths toward the comprehensive functional analysis of large gene lists. Nucleic Acids Res. 37(1), 1-13 (2009).

30. Benjamini Y, Hochberg Y. Controlling the false discovery rate: a practical and powerful approach to multiple testing. J. R. Statist. Soc. B 57, 289-300 (1995).

31. Roessler J, Ammerpohl O, Gutwein J, et al. Quantitative cross-validation and content analysis of the 450k DNA methylation array from Illumina, Inc. BMC Res. Notes 5, 210 (2012). 
32. Filer A. The fibroblast as a therapeutic target in rheumatoid arthritis. Curr. Opin. Pharmacol. 13(3), 413-419 (2013).

33. Marinova-Mutafchieva L, Taylor P, Funa K, Maini RN, Zvaifler NJ. Mesenchymal cells expressing bone morphogenetic protein receptors are present in the rheumatoid arthritis joint. Arthritis Rheum. 43(9), 2046-2055 (2000).

34. Terauchi R, Arai Y, Takahashi KA, et al. The effect of apoptosis signal-regulating kinase 1 gene transfer on rat collagen induced arthritis. J. Rheumatol. 32(12), 2373$2380(2005)$.

35. Mnich SJ, Blanner PM, Hu LG, et al. Critical role for apoptosis signal-regulating kinase 1 in the development of inflammatory $\mathrm{K} / \mathrm{BxN}$ serum-induced arthritis. Int. Immunopharmacol. 10(10), 1170-1176 (2010).

36. Philippe L, Alsaleh G, Pichot A, et al. miR-20a regulates ASK1 expression and TLR4-dependent cytokine release in rheumatoid fibroblast-like synoviocytes. Ann. Rheum. Dis. 72(6), 1071-1079 (2013).

37. Jones GC, Riley GP. ADAMTS proteinases: a multi-domain, multi-functional family with roles in extracellular matrix turnover and arthritis. Arthritis Res. Ther. 7(4), 160-169 (2005).

38. Martin JE, Alizadeh BZ, Gonzalez-Gay MA, et al. Identification of the oxidative stress-related gene MSRA as a rheumatoid arthritis susceptibility locus by genomewide pathway analysis. Arthritis Rheum. 62(11), 3183-3190 (2010).

39. Pauley KM, Satoh M, Chan AL, Bubb MR, Reeves WH, Chan EK. Upregulated miR-146a expression in peripheral blood mononuclear cells from rheumatoid arthritis patients. Arthritis Res. Ther. 10(4), R101 (2008). 
40. Stanczyk J, Pedrioli DM, Brentano F, et al. Altered expression of microRNA in synovial fibroblasts and synovial tissue in rheumatoid arthritis. Arthritis Rheum. 58(4), 1001-1009 (2008).

41. Kurowska-Stolarska M, Alivernini S, Ballantine LE, et al. MicroRNA-155 as a proinflammatory regulator in clinical and experimental arthritis. Proc. Natl. Acad. Sci. US A 108(27), 11193-11198 (2011).

42. Bluml S, Bonelli M, Niederreiter B, et al. Essential role of microRNA-155 in the pathogenesis of autoimmune arthritis in mice. Arthritis Rheum. 63(5), 1281-1288 (2011).

43. O’Connell RM, Kahn D, Gibson WS, et al. MicroRNA-155 promotes autoimmune inflammation by enhancing inflammatory T cell development. Immunity 33(4), 607$619(2010)$

44. Baer C, Claus R, Frenzel LP, et al. Extensive promoter DNA hypermethylation and hypomethylation is associated with aberrant microRNA expression in chronic lymphocytic leukemia. Cancer Res. 72(15), 3775-3785 (2012).

45. Vrba L, Munoz-Rodriguez JL, Stampfer MR, Futscher BW. miRNA gene promoters are frequent targets of aberrant DNA methylation in human breast cancer. PLoS One 8(1), e54398 (2013).

46. Rauch TA, Wu X, Zhong X, Riggs AD, Pfeifer GP. A human B cell methylome at 100-base pair resolution. Proc. Natl. Acad. Sci. U S A 106(3), 671-678 (2009).

47. Jjingo D, Conley AB, Yi SV, Lunyak VV, Jordan IK. On the presence and role of human gene-body DNA methylation. Oncotarget 3(4), 462-474 (2012). 
48. Ball MP, Li JB, Gao Y, et al. Targeted and genome-scale strategies reveal genebody methylation signatures in human cells. Nat. Biotechnol. 27(4), 361-368 (2009). 


\section{Financial disclosure/Acknowledgements}

The authors would like to thank Janet Turner for assistance with the collection of patient data. This work was supported by funding provided by the Haywood Rheumatism Research and Development Foundation. The authors have no conflict of interest to disclose and have relevant affiliations with or financial involvement with any organization or entity with a financial interest in or financial conflict with the subject matter or materials discussed in the manuscript. The authors did not receive any writing assistance in the preparation of the manuscript. 


\section{Figure legends}

Figure 1. Filtering criteria for the identification of CpGs differentially methylated between RA and OA fluid-derived FLS. The starting number of CpGs $(468,961)$ was derived through the removal of unreliable sites (CpGs with detection p-values $>0.05)$ and those with missing $\beta$-values in any one of the 11 samples, as described in the Methods. The horizontal dashed line after step 3 indicates implementation of a further filtering step as required for the identification of differentially methylated genes that were common with two other $450 \mathrm{~K}$ array studies (described in detail in the Results). Abbreviations: RA, rheumatoid arthritis; OA, osteoarthritis; FLS, fibroblast-like synoviocyte

Figure 2. Heatmap and clustering for the $328 \mathrm{CpGs}$ identified as differentially methylated between RA and OA fluid-derived FLS. DNA methylation across the 328 sites in each of the samples was analyzed by hierarchical clustering using the Euclidean distance and average linkage criteria. Each row represents an individual $\mathrm{CpG}$ site and each column a different sample (indicated beneath the heatmap). RA and OA-derived FLS are indicated by the red and green bars, respectively. Color gradation from yellow to blue represents low to high DNA methylation respectively, with $\beta$-values ranging from 0 (no methylation; yellow) to 1 (complete methylation; blue).

\section{Figure 3. Comparison of genes differentially methylated between RA and OA} fluid-derived FLS with those identified in tissue-derived FLS. A. Venn diagram comparison of genes identified as differentially methylated in this study (Step 3, Figure 1) with those reported to be differentially methylated in tissue-derived RA FLS in the 
studies of Nakano et al [15) and de la Rica et al [16). Genes identified as differentially methylated in those studies were obtained from Supplementary Table S1 and Supplementary Table 2, respectively, which accompany their published articles. Genes from our dataset that were common with one or both of these studies are indicated by light gray and dark gray shading, respectively. The overlap of 50 genes in common with Nakano et al [15), and 45 genes in common with de la Rica et al [16), was highly significant in each case $\left(p=1.62 \times 10^{-24}\right.$ and $p=1.03 \times 10^{-15}$, respectively; Hypergeometric Test based on Fisher's Exact Test). B. Heatmap and clustering of methylation data in this study for the 22 differentially methylated genes that were identified as common to all three studies (centre, Figure 3A). For each of three genes (KCNMA1, PKNOX2 and PRDM16), two separate CpGs were differentially methylated, totalling 25 unique CpGs in the heatmap presented. Each row represents an individual gene (with associated cg identifier in parentheses) and each column a different sample (indicated beneath the heatmap). RA and OA-derived FLS are indicated by the red and green bars, respectively. Color gradation from yellow to blue represents low to high DNA methylation respectively, with $\beta$-values ranging from 0 (no methylation; yellow) to 1 (complete methylation; blue).

Figure 4. Validation of candidate genes differentially methylated between RA and OA fluid-derived FLS. Bisulfite pyrosequencing was used to confirm 450K array methylation data for multiple candidate genes that were identified as A. common with (C18orf45, LMO4, MAP3K5, ODZ4, PKNOX2, PRDM16) or B. unique from (ADAMTS14, MIR155HG, RUNX1, ULK3) those genes identified in the studies of Nakano et al [15) and de la Rica et al [16). RA and OA fluid-derived FLS are depicted 
by circles and triangles respectively, and the short horizontal bar in each group indicates the mean value. Gene names are shown on the x-axis, and where for each gene methylation values are shown for the array (filled symbols; left) and pyrosequencing (open symbols; right).

Abbreviations: Pyro., bisulfite pyrosequencing.

Figure 5. Additional CpG sites in MAP3K5 and $R U N X 1$ that were differentially methylated between RA and OA fluid-derived FLS. Shown are additional CpGs adjacent to the array-identified site for A. MAP3K5 (one additional site), and B. RUNX1 (two additional sites), that were also found to be differentially methylated in RAderived FLS relative to OA FLS, as determined by pyrosequencing. Negative and positive base pair numbers on the $\mathrm{x}$-axis indicate bases upstream and downstream of the array $\mathrm{CpG}$ site, respectively. RA and OA fluid-derived FLS are depicted by circles and triangles respectively, and the short horizontal bar in each group indicates the mean. Abbreviations: bp, base pairs.

\section{Supplementary Figure 1. Heatmap and clustering for the 122 differentially} methylated genes that were unique to fluid-derived FLS. DNA methylation across the 122 genes (124 CpGs) in each of the samples was analyzed by hierarchical clustering using the Euclidean distance and average linkage criteria. Each row represents an individual $\mathrm{CpG}$ site and each column a different sample (indicated beneath the heatmap). RA and OA-derived FLS are indicated by the red and green bars, respectively. Color gradation from yellow to blue represents low to high DNA 
methylation respectively, with $\beta$-values ranging from 0 (no methylation; yellow) to 1 (complete methylation; blue).

\section{Supplementary Figure 2. Technical validation of $450 \mathrm{~K}$ array data by sodium}

bisulfite pyrosequencing. Correlation between array-derived methylation $\beta$-values and methylation levels quantified by bisulfite pyrosequencing was used to validate array data. One $\mathrm{CpG}$ in each of the ten differentially methylated genes presented in Figure 4 was examined in RA and OA fluid-derived FLS (90 discrete data points), represented as circles and crosses, respectively. Spearman's $r=0.955, \mathrm{p}<0.0001$.

\section{Supplementary Table 1. Assay details for candidate CpGs/genes interrogated by bisulfite pyrosequencing.}

*Further information that is not included here is available upon request. The horizontal dashed line between rows 9 and 10 separates candidate genes common with (above) and unique from (below) the studies of Nakano et al [15) and de la Rica et al [16).

$\dagger$ The prefix ' $b-$ ' denotes biotin labeling of the 5 ' end of the primer.

$\$$ The sequence indicated is post-bisulfite conversion and the letters ' $Y$ ' and ' $R$ ' denote the cytosine of interrogated $\mathrm{CpG}$ sites ('Y' and 'R' refer to sequencing along the upper and lower strands respectively).

Abbreviations: bp, base pairs

Supplementary Table 2. Complete list, $\beta$-value data and annotation for the 328 CpGs identified as differentially methylated between RA and OA fluid-derived FLS. 
*Unless otherwise stated, values are methylation array $\beta$-values. The solid black horizontal line between rows 260 and 261 separates CpGs that were hypomethylated (above) and hypermethylated (below) in RA fluid-derived FLS.

$\dagger$ FDR, false discovery rate adjustment for multiple comparisons [30).

Supplementary Table 3. Complete list, $\beta$-value data and annotation for the 122 differentially methylated genes (124 CpGs) that were unique to RA fluid-derived FLS.

*Unless otherwise stated, values are methylation array $\beta$-values. The solid black horizontal line between rows 95 and 96 separates CpGs that were hypomethylated (above) and hypermethylated (below) in RA fluid-derived FLS. $†$ FDR, false discovery rate adjustment for multiple comparisons [30). 